\title{
Mirroring Procedures for the Assessment of Asymmetry of Different Anatomical Structures of the Cranium: A Protocol Based on 3D-3D Superimposition
}

\author{
Daniele GIBELLI ${ }^{1}$, Valentina PUCCIARELLI ${ }^{1}$, Marina CODARI², \\ Luca PISONI ${ }^{1}$, Claudia DOLCl ${ }^{1}$, Chiarella SFORZA ${ }^{1}$ \\ 1. Dipartimento di Scienze Biomediche per la Salute, Università degli Studi di Milano, Milan, Italy; \\ 2. Unit of Radiology, IRCCS Policlinico San Donato, Via Morandi 30, San Donato Milanese, Milan, Italy \\ DOI: $10.15221 / 17.066 \quad$ http://dx.doi.org/10.15221/17.066
}

\begin{abstract}
The assessment of asymmetry has always represented one of the most relevant field of research in anatomy, with several applications in reconstructive and aesthetic surgery. This study aims at exposing an innovative protocol for the assessment of asymmetry of cranial structures based on 3D-3D superimposition and mirroring procedures.

Five patients who underwent a head CT scan were selected. Three anatomical structures were automatically segmented through ITK-SNAP free software: maxillary sinuses, zygomatic bones and the petrous portion of both temporal bones.

The left structure was then reflected automatically into the right image and superimposed over the contralateral one according to the least point-to-point distance on the entire surface. RMS (root mean square) distance was then automatically calculated for each superimposition. In addition, a chromatic map of superimposition was generated, showing the most variable areas between the two sides.

Possible statistically significant differences in RMS value according to the three anatomical structures were assessed through one-way ANOVA test $(p<0.05)$.

On average, RMS values for maxillary sinuses, zygomatic bones and petrous part of temporal bones were respectively $1.00 \mathrm{~mm}$ (SD: $0.27 \mathrm{~mm}), 0.70 \mathrm{~mm}$ (SD: $0.34 \mathrm{~mm}$ ) and $1.26 \mathrm{~mm}(\mathrm{SD}: 0.17 \mathrm{~mm})$. A significant difference among the symmetry of the three analysed structures was found $(p<0.01$, ANOVA).

A novel approach for the assessment of anatomical asymmetry is suggested, based both on a morphological and a quantitative evaluation of differences between the right and left side skull bones.
\end{abstract}

Keywords: Asymmetry, stereophotogrammetry, cranium, root mean square

\section{Introduction}

The study of asymmetry represents one of the most relevant fields of research in human anatomy, and finds applications in several fields of medical practice, among which reconstructive and aesthetic surgery is of relevant importance. In fact, the assessment of asymmetry provides a useful guideline for the reconstruction of anatomical structures, for example in cases of traumatic injuries. An example is provided by the reconstruction of the zygomatic bone, where the traumatized area is treated using the healthy counterlateral bony element as a model [1]. Therefore possible methods for assessing morphological and metrical characteristics of anatomical asymmetry represent an interesting field of research, not only from a theoretical point of view, but especially for practical reasons.

With time several methods for evaluating the characteristics of anatomical asymmetry in different districts have been developed, being the most commonly used the measurements and comparison of linear parameters between the right and the left side [2-5]. Measurements can be taken directly on dry bones through a caliper, or on CT-scans. The most important limits of this approach are the difficulties in reliably assessing landmarks for standardazying the reference distances [6] and the lack of a morphological assessment of surface asymmetry between the right and left side. A more precise approach to the quantification of asymmetry takes the procrustes elaboration of anatomical structures into consideration [7]: however, this type of approach fails in considering the 3D characteristics of surfaces.

In the last decade the development of novel methods of 3D image acquisition has enabled researchers to perform more detailed analyses of anatomical asymmetry, thanks to the chance of obtaining a 3D model of the structures of interest [8]: this novel improvement allowed operators to complete the metrical assesment of asymmetry through the calculation of volumes and surfaces [9]. In addition, the introduction of modern 3D image elaboration systems enables researchers to perform 3D-3D superimpositions: this approach may be applied also to the assessment of asymmetry of different 
anatomical structures, thanks to mirroring procedures which allow to reflect 3D models according to a plane of symmetry [1].

In this way the assessment of the entire surface of bones is easy to obtain: procedures of segmentation on CT-scans have already been used in surgery in order to obtain "mirrored" 3D models from the healthy portion of face, useful for the reconstruction of the fractured one [1]. For example, this approach is applied to zygomatic bones, in cases of delayed fractures with dislocation of bone fragments [10]. The 3D mirroring and registration procedure may provide innovative information concerning asymmetry through a morphological assessment of surface changes between the right and left side, and therefore add more data for a successful reconstruction.

This study aims at exposing a novel semi-automatic method for assessing and quantifying the asymmetry of different skull structures as depicted by TC scans. The method can be usefully applied to reconstructive and aesthetic surgery.

\section{Materials and methods}

Five patients who underwent a head CT scan were selected. The chosen CT-scans were assessed through ITK-SNAP software which allows to perform segmentation of structures of interest [11]. Three anatomical structures were semi-automatically segmented according to different grey levels: maxillary sinuses, zygomatic bones and the petrous portion of both temporal bones. As the above mentioned structures have a different density than the surrounding tissues, the segmentation was automatically performed by the software without the manual delimitation of surfaces of interest on each slide. The single manual interaction consists in identifying the beginning and end of the structure of interest within the CT scan volume. The corresponding 3D models from each patient were analysed through a 3D elaboration software (VAMC software, version 2.8.3, Canfield Scientific Inc.).

The left structure was then reflected automatically into the right image and superimposed over the contralateral one according to the least point-to-point distance on the entire surface. RMS (root mean square) distance was then automatically calculated for each superimposition. In addition, a chromatic sheet reproducing the discordant and concordant areas between the right and the mirrored left side was provided by the software (Fig. 1).
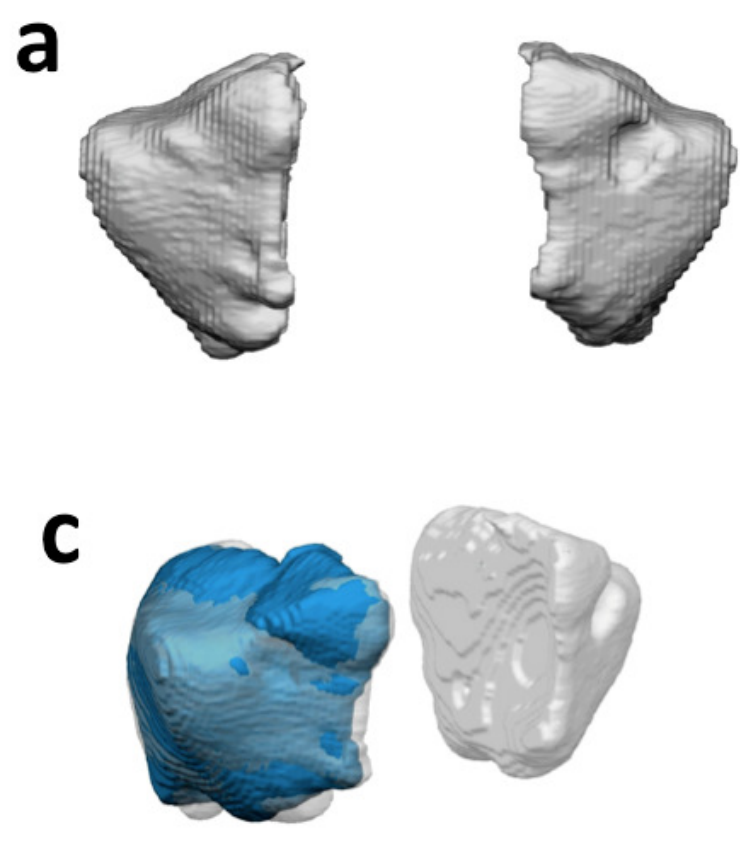
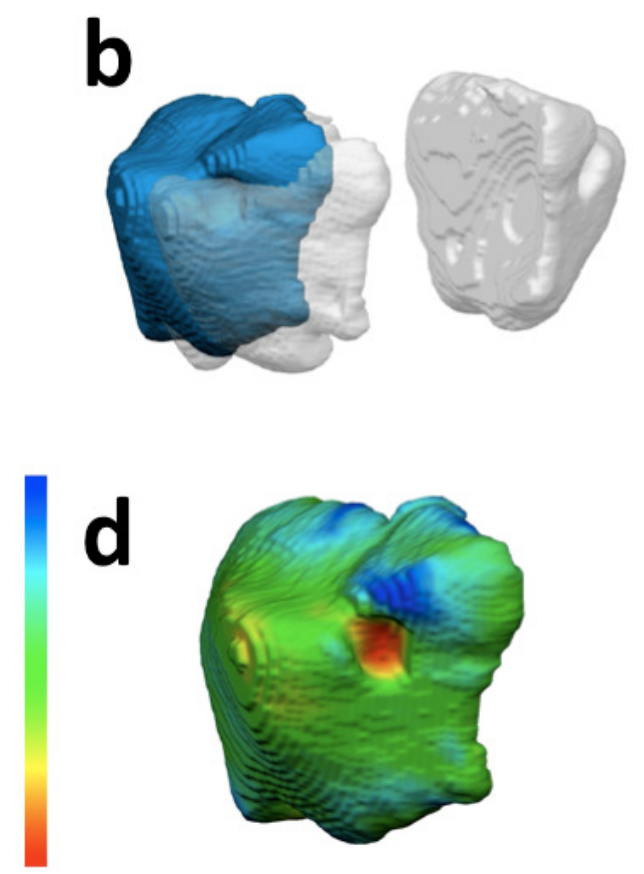

Fig. 1: steps for the assessment of asymmetry of maxillary sinuses: a) segmented $3 D$ volume of the sinuses (in frontal view); b) superimposition of the mirrored left one (in blue) on the right one (in transparency); c) automatic registration of the mirrored left sinus on the right one according to the least distance on the entire surface; $d$ ) calculation of RMS point-to-point distances: red, yellow and blue gradations show discordant regions between the two models (asymmetric areas), whereas green gradations indicate the concordant ones (symmetric areas) 
Notwithstanding the low manual interaction of the procedure, every step of segmentation, mirroring and registration was repeated by the same and another operator in order to test respectively intra- and inter-observer error: repeatability was assessed through Bland-Altman test for both parameters.

Possible statistically significant differences in RMS value according to the three anatomical districts were assessed through one-way ANOVA test $(p<0.05)$, followed by post-hoc comparisons.

Moreover, the volume of each structure was automatically calculated through the previously cited 3D image elaboration software: an asymmetry index, defined as the absolute value of the following formula: (volume right side - volume left side) ${ }^{\star} 100 /$ volume right side, was computed. Two-way ANOVA test was applied in order to verify possible statistically significant differences according to side and bone structure $(p<0.05)$.

\section{Results}

Maxillary sinuses showed the highest repeatability (respectively $90 \%$ and $88 \%$ for intra- and inter-observer error), followed by the zygomatic bones ( $79 \%$ for both the parameters) and the petrous part of temporal bone (respectively $74 \%$ and $73 \%$ ).

On average, the RMS value for maxillary sinuses was $1.00 \mathrm{~mm}$ (SD: $0.27 \mathrm{~mm}$ ); zygomatic bones reached an RMS value of $0.70 \mathrm{~mm}$ (SD: $0.34 \mathrm{~mm}$ ). The highest surface variability between the right and the left side was shown by the petrous part of temporal bone, with an RMS of $1.26 \mathrm{~mm}$ (SD: 0.17 ) (Fig. 2).

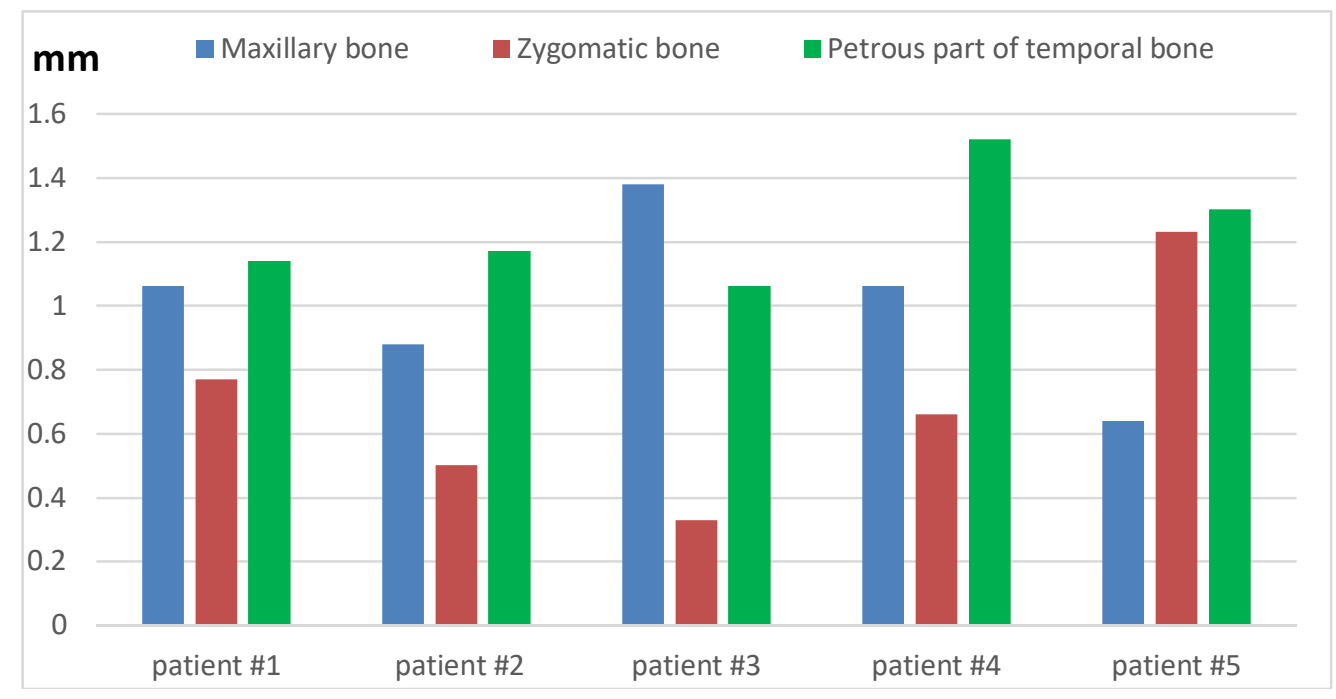

Fig. 2: RMS values for the five subjects divided according to the type of anatomical structure. Patient\#1: male 42 years old; Patient\#2: male 30 years old; Patient\#3: male 34 years old; Patient\#4: female 36 years old; Patient\#5: female 32 years old.

In addition, the registration procedure was able to detect areas of discordance through a chromatic map where red, yellow and blue gradations show discordant (asymmetric) regions between the virtual models of the two body sides, whereas green regions are the most symmetric (Fig. 3).
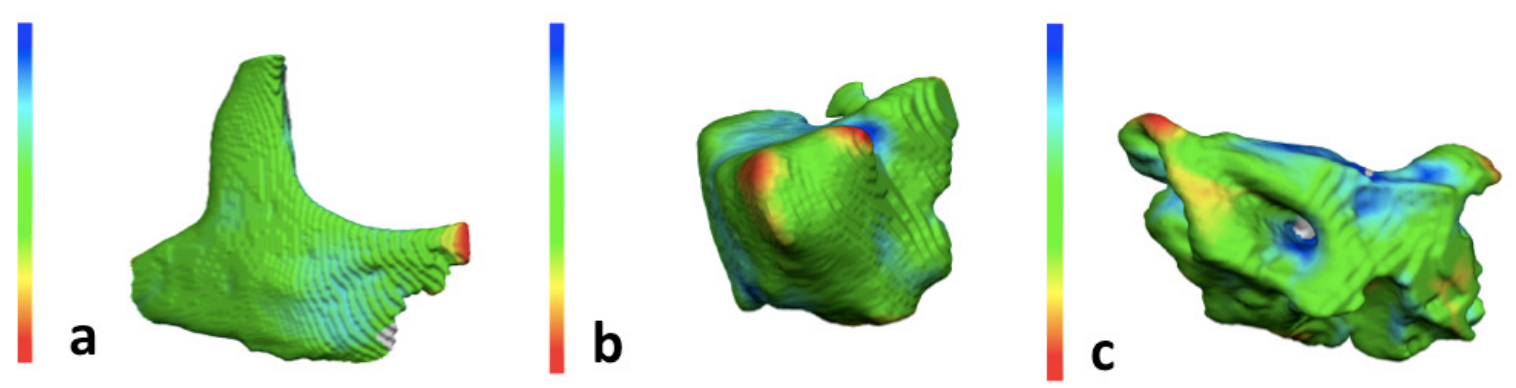

Fig. 3: examples of the results of registration and calculation of point-to-point distances between the right and the mirrored left side of a) zygomatic bone; b) maxillary sinus; c) petrous part of the temporal bone. Red, yellow and blue gradations show discordant regions between the two models (asymmetric areas), whereas green gradations depict the concordant parts (symmetric areas) 
Volumes of anatomical structures are shown in Table 1: the left side highlighted a prevalence in most of cases. Statistically significant differences in volume were found according to bone (F: 15.4; P: $0.0001)$, not according to side (F: $1 ; P: 0.3273)$. No interaction was found (F: 0.01; P: 0.9901).

\begin{tabular}{|c|c|c|c|c|}
\hline \multicolumn{2}{|c|}{} & Maxillary bone & Zygomatic bone & $\begin{array}{c}\text { Petrous part of the } \\
\text { temporal bone }\end{array}$ \\
\hline \multirow{2}{*}{ Right side } & Mean & 12.82 & 6.25 & 6.17 \\
\cline { 2 - 5 } & SD & 3.52 & 1.63 & 2.93 \\
\hline \multirow{2}{*}{ Left side } & Mean & 13.75 & 7.28 & 7.52 \\
\cline { 2 - 5 } & SD & 4.02 & 2.38 & 3.01 \\
\hline
\end{tabular}

Table 1: mean volumes of different anatomical structures on the right and left side: all measurements are expressed in $\mathrm{cm}^{3} ; \mathrm{SD}$ : standard deviation

The highest asymmetry index was observed in the petrous portion of temporal bone (34\%; SD: $20 \%$ ), followed by zygomatic bone (16\%; SD: $15 \%$ ) and the maxillary sinuses (10\%; SD: $8 \%$ ): results for each subject are shown in Figure 4.

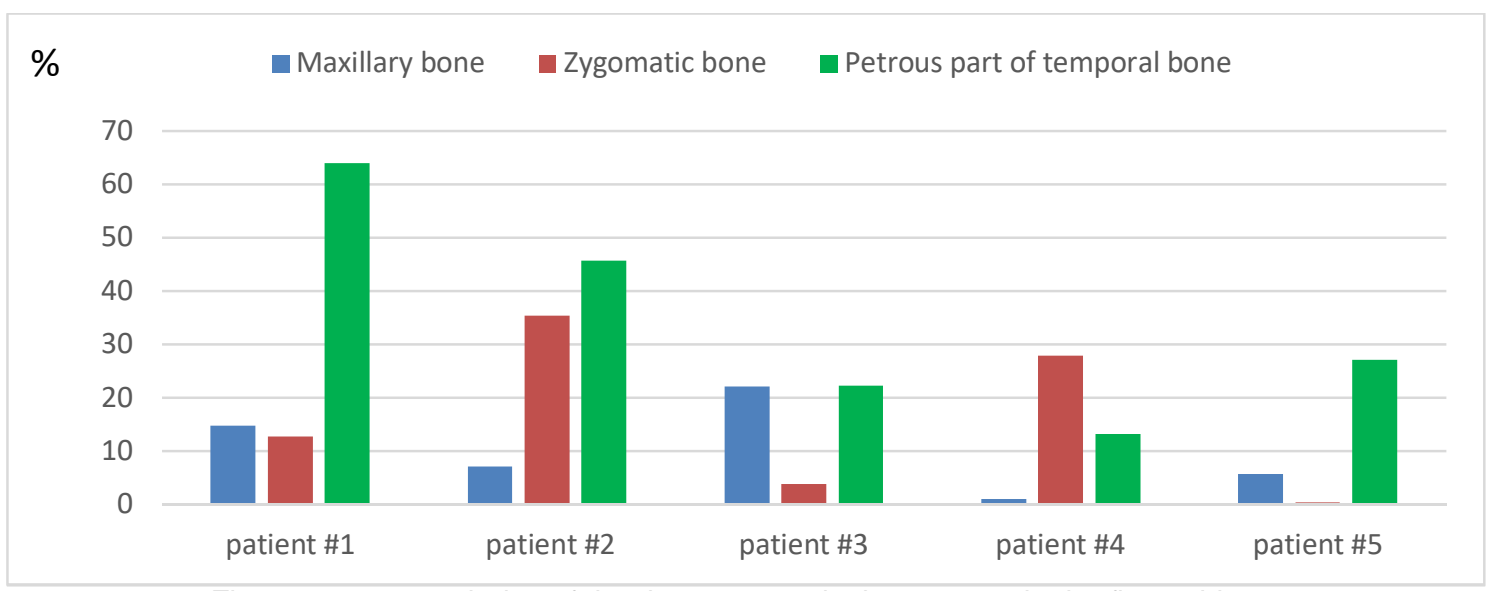

Fig. 4: asymmetry index of the three anatomical structures in the five subjects

A significant difference among the symmetry of the three analysed structures was found $(p<0.01)$; post-hoc comparisons found that RMS values from the petrous portion of temporal bone were significantly higher than those obtained for the zygomatic bone, whereas no statistical difference was found between asymmetry of maxillary sinuses with the other two districts.

\section{Discussion}

Symmetry is a relevant biological characteristic of anatomical structures and has several consequences in clinical and surgical practice. The traditional approach based on linear measurements can give only a partial indication about symmetry, as long as it considers the change in position of landmarks and does not provide a 3D surface analysis of differences between the right and the left side.

The introduction of modern 3D image acquisition and elaboration procedures allows the operators to perform more precise and complete analyses based on mirroring and registration techniques. This type of procedures have already been applied to the assessment of facial asymmetry, through the reconstruction of a midsagittal plane [12-14].

In the last years the introduction of automatic and manual segmentation on CT-scans has allowed the operators to easily obtain 3D models of bony structures and to reproduce the same methodology for assessing asymmetry [15].

This article aimed at testing this procedure to three different bony structures segmented from CT-scan. The most important advantages concern the assessment of surface differences through a metrical parameter (RMS value) and a morphological analysis through the chromatic map of modifications, automatically provided by the software. In addition, this technique allows to overcome the traditional limits encountered by literature, such as the determination of the midsagittal plane for the mirroring procedure. In fact, most of literature considers a midsagittal plane generated by midfacial anatomic landmarks [16,17]. Other more innovative techniques are based on the determination of a midsagittal 
plane according to the registration between the 3D model of cranium and its "mirrored" version [6]. The collocation of a midsagittal plane involves relevant difficulties as there are no universally accepted midpoints to guide a comparison between the two sides of the skull and manual methods for collocating a symmetry plane are affected by low reliability [18].

The present procedure solves this problem as the mirroring procedure is automatically performed by the software, reducing the possible error due to manual localization of the midsagittal plane. In addition, the entire procedure proved to be well repeatable.

In conclusion, this article proposes a novel approach to the assessment of asymmetry of bony structures based on mirroring and registration of segmented 3D models from CT scans. The method may be used to study anatomical symmetry and for planning reconstructive surgery in case of previous traumatic injuries or a tumour removal.

\section{References}

[1] F. Feng, et al. "Mirror imaging and preshaped titanium plates in the treatment of unilateral malar and zygomatic arch fractures", Oral Surg Oral Med Oral Pathol Oral Radiol Endod Vol. 112, 2011, pp. 188-94, http://dx.doi.org/10.1016/j.tripleo.2010.10.014 .

[2] F.N. Fries et al. "Comparing the left and right Whitnall's tubercles and their relation to the frontozygomatic suture. Application to symmetry following lateral orbital surgery", Orbit Vol. 35, 2016, pp. 305-8.

[3] Y.L. Zhang et al. "Morphological analysis of the temporomandibular joint between patients with facial asymmetry and asymptomatic subjects by 2D and 3D evaluation", Medicine Vol. 95, 2016, pp. e3052-8, http://dx.doi.org/10.1097/MD.0000000000003052 .

[4] A.R. Sepahdari, S. Mong "Skull base CT: normative values for size and symmetry of the facial nerve canal, foramen ovale, pterygoid canal, and foramen rotundum", Surg Radiol Anat Vol. 35, 2013, pp. 19-24, http://dx.doi.org/10.1007/s00276-012-1001-4 .

[5] T. Lepich, et al. "Female and male orbit asymmetry: digital analysis", Adv Clin Exp Med Vol. 26, 2017, pp. 69-76, http://dx.doi.org/10.17219/acem/58783 .

[6] X. Gong et al., "Quantification of zygomatic complex symmetry using 3-dimensional computed tomography", J Oral Maxillofac Surg Vol. 72, 2014, pp. 2053.e1-e8, http://dx.doi.org/10.1016/i.joms.2014.06.447.

[7] $\mathrm{H}$. Jung et al. "A comparison of cranial fluctuating asymmetry between the two sexes in a Joseon Dynasty population of Korea", Anthropol Anz Vol. 73, 2017, pp. 215-33, http://dx.doi.org/10.1127/anthranz/2016/0585.

[8] D. Gibelli et al. "An Assessment of How Facial Mimicry Can Change Facial Morphology: Implications for Identification", J Forensic Sci Vol. 62, No. 2, 2017, pp. 405-10, http://dx.doi.org/10.1111/1556-4029.13295.

[9] H. Lin, et al. "Mandibular asymmetry: a three-dimensional quantification of bilateral condyles", Head \& Face Med Vol. 9, 2013, pp. 42-8, http://dx.doi.org/10.1186/1746-160X-9-42 .

[10] Y. He et al. "Zygomatic surface marker-assisted surgical navigation: a new computer-assisted navigation method for accurate treatment of delayed zygomatic fractures", J Oral Maxillofac Surg Vol. 71, 2013, 2101-14, http://dx.doi.org/10.1016/i.joms.2013.07.003 .

[11] P.A. Yushkevich et al. "User-guided 3D active contour segmentation of anatomical structures: significantly improved efficiency and reliability", Neuroimage Vol. 31, 2006, pp. 1116-28.

[12] V.F. Ferrario, et al., "The effect of sex and age on facial asymmetry in healthy subjects: a cross-sectional study from adolescence to mid-adulthood", J Oral Maxillofac Surg Vol. 59, 2001, pp. 382-8, http://dx.doi.org/10.1053/joms.2001.21872.

[13] M. Codari et al., "Facial thirds-based evaluation of facial asymmetry using stereophotogrammetric devices: application to facial palsy subjects", J Craniomaxillofac Surg Vol. 45, 2017, pp. 76-81, http://dx.doi.org/10.1016/i.jcms.2016.11.003 .

[14] P. Berssenbrugge et al. "2D and 3D analysis methods of facial asymmetry in comparison", J Craniomaxillofacial Surg Vol. 42, 2014, pp. e327-334, http://dx.doi.org/10.1016/i.jcms.2014.01.028 .

[15] S. Pirner, et al. "CT-based manual segmentation and evaluation of paranasal sinuses" Eur Arch Otorhinolaryngo/ Vol. 266, 2009, pp. 507-18, http://dx.doi.org/10.1007/s00405-008-0777-7 .

[16] L.G. Farkas, G. Cheung, "Facial asymmetry in healthy North American Caucasians. An anthropometrical study", Angle Orthod Surg Vol. 51, 1996, pp. 70-7.

[17] E. Ellis 3rd, W. Kittidumkerng, "Analysis of treatment for isolated zygomaticomaxillary complex fractures", J Oral Maxillofac Surg Vol. 54, 1996, pp. 386-400.

[18] G. Roumeliotis, et al., "Application of a novel semi-automatic technique for determining the bilateral symmetry plane of the facial skeleton of normal adult males", J Craniofac Surg Vol. 26, 2015, pp. 1997-2001, http://dx.doi.org/10.1097/SCS.0000000000001937. 